\title{
Changes of Serum Insulin-like Growth Factor-2 Response to Negative Symptom Improvements in Schizophrenia Patients Treated with Atypical Antipsychotics*
}

\author{
Xue-lin $\mathrm{CHAO}^{1 \dagger}$, Shu-zhen JIANG ${ }^{2 \dagger}$, Jian-wen $\mathrm{XIONG}^{3}$, Jin-qiong $\mathrm{ZHAN}^{2}$, Bo $\mathrm{WEI}^{2,3}$, Chun-nuan $\mathrm{CHEN}^{4 \#,}$ \\ Yuan-jian $\mathrm{YANG}^{2,3 \#}$ \\ ${ }^{I}$ Department of Psychosomatic Medicine, The First Affiliated Hospital of Nanchang University, Nanchang 330006, China \\ ${ }^{2}$ Biological Psychiatry Laboratory, Jiangxi Mental Hospital/Affiliated Mental Hospital of Nanchang University, Nanchang \\ 330029, China \\ ${ }^{3}$ Department of Psychiatry, Jiangxi Mental Hospital/Affiliated Mental Hospital of Nanchang University, Nanchang 330029, \\ China \\ ${ }^{4}$ Department of Neurology, The Second Clinical Medical College, The Second Affiliated Hospital, Fujian Medical University, \\ Quanzhou 362000, China
}

(C) The Author(s) 2020, corrected publication October 2020

\begin{abstract}
Summary: Accumulating evidence suggests that a disruption of early brain development, in which insulin-like growth factor-2 (IGF-2) has a crucial role, may underlie the pathophysiology of schizophrenia. Our previous study has shown that decreased serum IGF-2 was correlated with the severity of psychopathology in patients with schizophrenia. Here we conducted a prospective observation trial to investigate the effects of atypical antipsychotics on serum IGF-2 level and its relationship with clinical improvements in schizophrenia patients. Thirty-one schizophrenia patients with acute exacerbation and 30 healthy individuals were recruited in this study. Psychiatric symptoms were assessed using the Positive and Negative Syndrome Scale (PANSS) and serum IGF-2 levels were determined using ELISA. We found that schizophrenia patients with acute exacerbation had lower serum IGF-2 levels than control individuals at baseline $(P<0.05)$. After 2 months of atypical antipsychotic treatment, a significant improvement in each PANSS subscore and total score was observed in patients (all $P<0.01$ ), and the serum IGF-2 levels of patients were significantly increased compared with those at baseline $(203.13 \pm 64.62 v s .426 .99 \pm 124.26 \mathrm{ng} / \mathrm{mL} ; t$ $=-5.044, P<0.001)$. Correlation analysis revealed that the changes of serum IGF-2 levels in patients were significantly correlated with the improvements of negative symptoms $(r=-0.522, P=0.006)$. Collectively, our findings demonstrated changes of serum IGF-2 response to improvements of negative symptoms in schizophrenia patients treated with atypical antipsychotics, suggesting that serum IGF-2 might be a treatment biomarker for schizophrenia.

Key words: schizophrenia; atypical antipsychotic; insulin-like growth factor-2; negative symptoms
\end{abstract}

Schizophrenia is a chronic and severe mental disorder with approximately $1 \%$ prevalence in the worldwide population. The symptoms of schizophrenia generally comprise three categories: positive symptoms,

The original version of this article was revised due to a retrospective Open Access order.

Xue-lin CHAO, E-mail: ljz16888@163.com; Shu-zhen JIANG, E-mail: 641506002@qq.com

${ }^{\dagger}$ The authors contributed equally to this study.

\#Corresponding authors, Chun-nuan CHEN, E-mail: chenchunnuan1983@aliyun.com; Yuan-jian YANG, E-mail: yuanjimyang@yeah.net

*This work was supported by grants from the National Natural Science Foundation of China (No. 81760254), the Natural Science Foundation of Fujian Province of China (No. 2019J01164) and the Scientific Foundation of Quanzhou City for High Level Talents (No. 2019C075R). negative symptoms and cognitive impairments ${ }^{[1]}$. The causes of schizophrenia are complex and are not fully understood, so current treatments focus on managing symptoms and solving problems related to day-to-day functioning ${ }^{[2,3]}$.

Antipsychotic medications are the primary treatment strategy for schizophrenia ${ }^{[3]}$. First-generation antipsychotics (also known as typical antipsychotics) have a definitely therapeutic effect for positive symptoms, while have little or no effect on the negative or cognitive symptoms ${ }^{[4]}$. Second-generation drugs (SGAs), which are known as atypical antipsychotics, have been developed and introduced clinically in the 1970s. Atypical antipsychotics represent a new generation of antipsychotics with low extrapyramidal side-effects (EPS) and lack of sustained prolactin elevation ${ }^{[5]}$. They have shown superiority to typical 
antipsychotics in improving negative symptoms and cognitive dysfunction ${ }^{[5,6]}$. Recommended therapeutic doses for atypical antipsychotics confer a greater improvement in negative symptoms compared with haloperido[ ${ }^{[7]}$. Plenty of clinical studies have demonstrated that atypical antipsychotics could significantly improve the cognitive function of schizophrenia patients ${ }^{[8,}$ 9]. Therefore, atypical antipsychotic drugs have been more frequently used for the management of schizophrenia in recent years.

Neurodevelopmental hypothesis of schizophrenia proposes that schizophrenia is the behavioral outcome of an aberration in brain development processes during early life ${ }^{[10]}$. Numerous studies in areas of epidemiology, genetics and neuroimaging have provided strong evidence for this hypothesis ${ }^{[11,12]}$. Insulin-like growth factors (IGFs) are members of the insulin superfamily and have an essential role in the brain development ${ }^{[13]}$. As one of the IGFs, IGF-2 is expressed in brains not only during the development period but also during adulthood $^{[14]}$. Recent studies have demonstrated that alterations of IGF-2 signaling are implicated in the pathogenesis of schizophrenia ${ }^{[15-18]}$. Specifically, a large CommonMind consortium RNA-sequencing study revealed that IGF-2 was the top downregulated gene in the prefrontal cortex of patients with schizophrenia ${ }^{[15]}$. The methylation levels of an enhancer within the IGF-2 gene in isolated neurons from the prefrontal cortex were significantly decreased in schizophrenia patients ${ }^{[16]}$. Serum levels of IGF-2 were significantly increased in male Arab schizophrenia patients with stable dose of oral antipsychotic medication ${ }^{[17]}$. Our previous study found that serum IGF-2 levels were much lower than those of controls, and decreased IGF-2 levels were correlated with the severity of negative symptoms ${ }^{[18]}$. However, whether atypical antipsychotics treatment would affect the levels of serum IGF-2 in schizophrenia patients remains unknown.

In this study, we conducted a prospective observation trial to investigate the effects of atypical antipsychotics on serum IGF-2 level and its relationship with clinical improvements in schizophrenia patients. We hypothesized that peripheral IGF-2 level might be a potential treatment biomarker for schizophrenia.

\section{MATERIALS AND METHODS}

\subsection{Subjects}

Thirty-one inpatients diagnosed with schizophrenia were recruited from Jiangxi Mental Hospital. All these recruited patients had acute exacerbation for their psychotic symptoms and were admitted into acute ward for treatment. They were diagnosed by two trained research psychiatrists according to the Structured Clinical Interview for Diagnostic and Statistical Manual of Mental Disorders, Fourth Edition (DSM-IV), and were required to meet the following conditions: (1) The age was between 18 and 50 years old; (2) the Positive and Negative Syndrome Scale (PANSS) score of patients was between 60 and 120 at the baseline interview; (3) the patients were not diagnosed with any other DSM-IV axis I or axis II disorders other than schizophrenia. Patients with current pregnancy, neurological disorders, serious somatic disorders, and drug/alcohol abuse or addiction were excluded from the study.

Thirty healthy individuals were recruited from the local community and entered into the healthy control group. The controls were matched with the patients by age, gender, education, and body mass index (BMI). Subjects with an individual or family history of psychiatric disorders or drug abuse were excluded from the control group.

The research was conducted in accordance with the Declaration of Helsinki and other relevant national and international regulations. The procedures of this study were reviewed and approved by the Institutional Review Board at Jiangxi Mental Hospital. A written informed consent was obtained from each of the patients and the controls or their legal guardians before start of this study.

\subsection{Medication and Clinical Assessments}

The type of atypical antipsychotics for patients was selected according to their demographic and clinical symptom characteristics. Atypical antipsychotics included in this study consisted of risperidone $(n=10)$, aripiprazole $(n=7)$, olanzapine $(n=6)$ and clozapine $(n=8)$. The patients treated with clozapine met the criteria for refractory schizophrenia. These recruited patients only received a single atypical antipsychotic during the study period. Benzene hydrochloride was used only when the patient had significant extrapyramidal response. According to several treatment guidelines for schizophrenia ${ }^{[19,20]}$, the duration of sufficient treatment is approximately 6-10 weeks in the acute phase of treatment. Clinically, the most commonly used treatment time is 8 weeks for patients with schizophrenia in the acute phase. In this study, only those patients who completed an 8-week follow-up were included in the present study.

The severity of psychiatric symptoms of patients was assessed at baseline and followed up using the positive and negative symptoms scale (PANSS). The evaluation was performed by two psychiatrists who had been trained in the consistency of use of PANSS before the study began. The interobserver correlation coefficient for the PANSS total score was $>0.80$.

\subsection{Measurement of Serum IGF-2 Level}

Blood samples were collected from all the subjects between 7 am and 9 am after overnight fasting. After centrifugation of blood, we separated the serum, aliquoted the samples and immediately stored them at 
$-80^{\circ} \mathrm{C}$. The content of serum IGF-2 was determined by using ELISA with available kits on the market (Catalog \# SEA051Hu; Wuhan USCN Business, China). The sensitivity for IGF-2 was $0.264 \mathrm{ng} / \mathrm{mL}$, with variation coefficients of inter- and intra-assay of $10 \%, 12 \%$, respectively. Briefly, the same volume of standard or serum sample was added to the microplate well with a biotin-binding antibody that specifically binds to IGF2. Then, Avidin conjugated to Horseradish Peroxidase (HRP) was added to each microplate well and incubated. After TMB substrate solution was added, only those wells containing IGF-2, biotin-conjugated antibody and enzyme-conjugated Avidin would display a change in color. We stopped the reaction of the enzyme with the substrate by adding a solution of sulfuric acid and measured the color change at a wavelength of $450 \mathrm{~nm}$ using Spectrophotometer. Each sample was determined in duplicate. A standard curve was constructed by plotting the mean absorbance $(A)$ and concentration for each standard and a best fit curve was drawn through the points on the graph. The concentration of IGF-2 in the sample was then calculated by comparing the $A$ of the sample to the standard curve. An investigator who was blind to the clinical grouping was assigned to analyze all samples. To prevent inter-assay variance, we analyzed all the samples in the same assay.

\subsection{Statistical Analysis}

Demographic characteristics were compared between the control and the patient groups using Student's $t$-test for quantitative variables and chisquared test for qualitative variables. To compare the group difference in IGF-2 levels between controls and patients, ANCOVA was conducted using age, gender, education years, and BMI as covariates to minimize the potential effect of these factors on IGF-2 expression. A paired $t$-test was used to compare PANSS total and subscores as well as serum IGF-2 concentrations between two time points (at baseline and 8th week after antipsychotic treatment) in the same patients. KruskalWallis test was adopted to assess whether there were differences in psychiatric symptoms and IGF-2 levels among different atypical antipsychotics at the endpoint. Spearman's correlation analysis was used to assess the correlation between change of PANSS scores and IGF2 levels in patient group. All statistical tests were twotailed, and the level of statistical significance was set at $P<0.05$.

\section{RESULTS}

\subsection{Demographic and Clinical Characteristics and Serum IGF-2 Measurement}

This is an open-label prospective observational study. Thirty-one schizophrenia patients who completed an 8-week follow-up were enrolled in this study. The demographic and clinical characteristics of the participants included in the analysis are shown in table 1. There were no significant differences in age, gender, education years, or BMI between the patient groups and the control group (all $P>0.05$ ). Then the levels of serum IGF-2 were measured in both groups. As we previously reported ${ }^{[18]}$, schizophrenia patients showed much lower levels of serum IGF-2 than the healthy controls $(203.13 \pm 64.62$ vs. $442.34 \pm 105.33 \mathrm{ng} /$ $\mathrm{mL} ; F=20.702, P<0.001)$. ANCOVA analysis revealed that the group difference of serum IGF-2 was still significant $(F=4.393, P=0.002)$ when the variables of age, gender, education years, and BMI were included as covariates (table 1 ).

\subsection{Changes of PANSS Scores and Serum IGF- 2 Levels in Patients after Atypical Antipsychotic Treatment}

The primary endpoint was the changes of PANSS scores from baseline to the end of 8 weeks. The number of patients treated with risperidone, aripiprazole, olanzapine and clozapine was 10 , 7, 6 and 8, respectively. The clinical efficacy of atypical antipsychotics in schizophrenia patients with acute exacerbation is shown in table 2. There was a significant improvement in each PANSS subscore and

Table 1 Baseline demographic and clinical characteristics and IGF-2 levels for the two groups

\begin{tabular}{lcccc}
\hline Items & Controls $(n=30)$ & Patients $(n=31)$ & $t / \chi^{2} / F$ & $P$ value \\
\hline Age (years) & $34.23 \pm 9.31$ & $30.26 \pm 8.48$ & 1.745 & 0.086 \\
Gender (M/F) & $13 / 17$ & $16 / 15$ & 0.419 & 0.517 \\
Education (years) & $12.57 \pm 4.48$ & $11.84 \pm 3.76$ & 0.689 & 0.494 \\
BMI $\left(\mathrm{kg} / \mathrm{m}^{2}\right)$ & $21.19 \pm 1.67$ & $21.77 \pm 2.21$ & -1.156 & 0.252 \\
Illness duration (years) & $\mathrm{NA}$ & $5.93 \pm 5.57$ & - & - \\
IGF-2 level (ng/mL) & $442.34 \pm 105.33$ & $203.13 \pm 64.62$ & 4.393 & 0.002 \\
\hline
\end{tabular}

Table 2 Comparison of PANSS scores and IGF-2 levels between baseline and endpoint in patients

\begin{tabular}{lccrc}
\hline & Baseline & Endpoint & $t$ & $P$ value \\
\hline PANSS & $22.84 \pm 3.95$ & $7.89 \pm 2.33$ & 22.389 & $<0.001$ \\
Positive subscore & $15.48 \pm 5.28$ & $7.46 \pm 0.64$ & 8.249 & $<0.001$ \\
Negative subscore & $44.74 \pm 5.90$ & $20.61 \pm 6.20$ & 26.555 & $<0.001$ \\
General subscore & $80.03 \pm 10.44$ & $36.25 \pm 9.41$ & 30.540 & $<0.001$ \\
Total scores & $203.13 \pm 64.62$ & $426.99 \pm 124.26$ & -5.044 & $<0.001$ \\
IGF-2 level (ng/mL) & $442.34 \pm 105.33$ & $203.13 \pm 64.62$ & 4.393 & 0.002 \\
\hline
\end{tabular}


total score when the patients completed the research (Paired $t$-tests; all $P<0.01$ ). Then a non-parametric test (Kruskal-Wallis test) was conducted to find out if there were any differences in psychiatric improvements among different atypical antipsychotic treatments. As shown in table 3, there were no significant differences in each PANSS subscore and total score among risperidone, aripiprazole, olanzapine and clozapinetreated groups at the endpoint $\left(\chi^{2}=3.844, P=0.279\right)$.

Along with clinical improvement, serum IGF-2 levels after monotherapy with atypical antipsychotic for 8 weeks were significantly increased as compared with baseline $(203.13 \pm 64.62 v s .426 .99 \pm 124.26 \mathrm{ng} / \mathrm{mL}$; $t=-$ 5.044, $P<0.001$ ) (table 2 and fig. 1). The level of serum IGF-2 in patients after 8-week atypical antipsychotic monotherapy $(426.99 \pm 124.26 \mathrm{ng} / \mathrm{mL})$ was comparable with the level in the controls $(442.34 \pm 185.33 \mathrm{ng} /$ $\mathrm{mL})(P>0.05)$. All these antipsychotics could induce a significant increase in the levels of serum IGF-2 $(P$ $<0.05$ ), and no significant difference was observed among the groups in the serum IGF-2 level at the endpoint of study (Kruskal-Wallis test, $\chi^{2}=2.699, P=$ 0.440) (table 3).

\subsection{Relationship between Negative Symptom} Improvement and Change of Serum IGF-2 Levels

Spearman's correlation analysis was performed to explore whether the changes of serum IGF-2 levels were associated with the improvements of psychiatric symptoms in the patients. We found that the increase of serum IGF-2 had no relationship with the change of PANSS positive subscores $(r=-0.054, P=0.793)$,

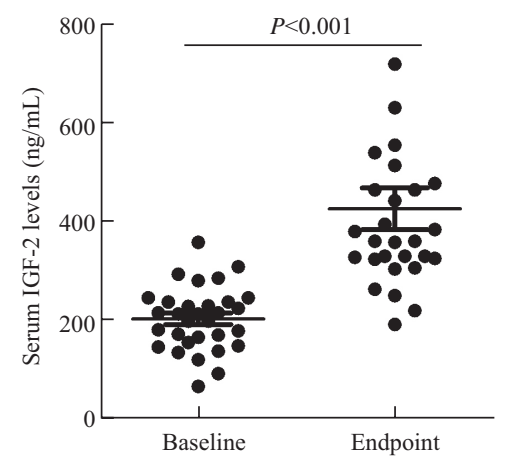

Fig. 1 Serum IGF-2 levels of schizophrenia patients with acute exacerbation at baseline and endpoint (8th-week)

Scatter plots for the comparison of serum IGF-2 levels at baseline and endpoint. The sample means are indicated by the black bars. general psychopathological subscores $(r=-0.051, P=$ $0.792)$, or total scores $(r=-0.340, P=0.089)$, but was significantly correlated with the reduction of negative subscores $(r=-0.522, P=0.006)$ in the patients treated with atypical antipsychotic (fig. 2).

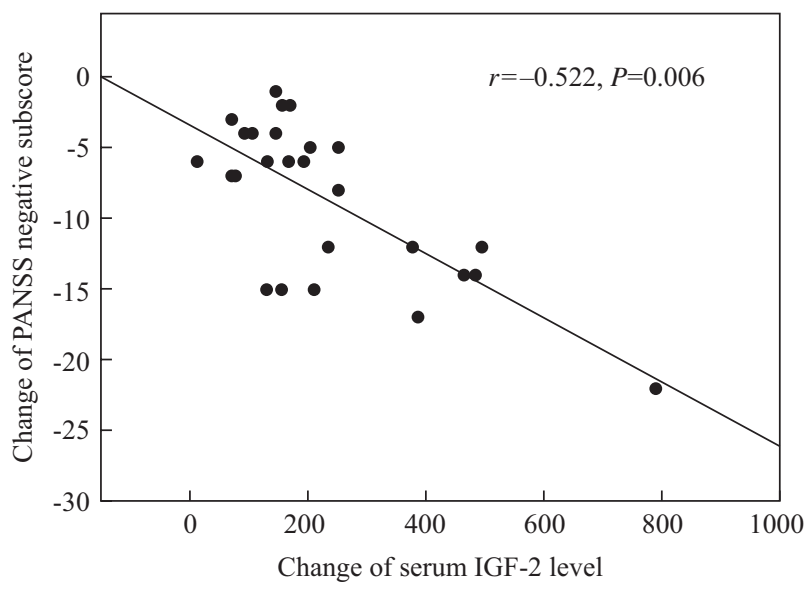

Fig. 2 The correlation between the change of serum IGF-2 levels and improvement of negative symptoms in patients with atypical antipsychotic treatment

\section{DISCUSSION}

This is an observational study aiming to investigate the treatment responses of atypical antipsychotics on psychiatric symptoms and serum IGF-2 levels in patients with schizophrenia. We found that serum levels of IGF-2 were significantly lower in the schizophrenia patients than in the healthy controls. Treatment with atypical antipsychotics for 8 weeks significantly improved psychiatric symptoms, in parallel with increased serum IGF-2 levels in patients. The increase in IGF-2 levels in patients was significantly correlated with the improvement of negative symptoms. This is the first study related to serum IGF-2 levels and treatment response of psychiatric symptoms.

Positive symptoms, negative symptoms and cognitive impairments are the three core symptoms of schizophrenia ${ }^{[1]}$. Positive symptoms indicate anomalous patterns of internal brain activity, including altered perceptions, abnormal thinking, and whimsical behaviors ${ }^{[2]}$. Negative symptoms comprise loss of motivation, social withdrawal, difficulty in showing emotions, and lack of enjoyment in daily

Table 3 Serum IGF-2 level in schizophrenia patients before and after treatment

\begin{tabular}{|c|c|c|c|c|}
\hline & \multicolumn{2}{|c|}{ IGF-2 level (ng/mL) } & \multirow{2}{*}{$t$} & \multirow{2}{*}{$P$ value } \\
\hline & Baseline & Endpoint & & \\
\hline Risperidone $(n=10)$ & $215.77 \pm 74.52$ & $344.30 \pm 126.44$ & -2.769 & 0.022 \\
\hline Aripiprazole $(n=7)$ & $207.51 \pm 66.55$ & $413.58 \pm 93.41$ & -2.629 & 0.039 \\
\hline Olanzapine $(n=6)$ & $226.76 \pm 12.20$ & $397.92 \pm 95.19$ & -5.367 & 0.006 \\
\hline Clozapine $(n=8)$ & $190.89 \pm 46.55$ & $366.17 \pm 44.60$ & -12.860 & $<0.001$ \\
\hline$\chi^{2}$ & 1.466 & 2.699 & & \\
\hline$P$ value & 0.690 & 0.440 & & \\
\hline
\end{tabular}


activities $^{[22]}$. Cognitive deficits, including impairments of processing speed, attention, working memory and execute function, generally emerge before positive and negative symptoms and persist throughout the illness's course $^{[23]}$. Treatment with atypical antipsychotics could significantly improve global clinical efficacy in patients with schizophrenia ${ }^{[24-26]}$. In current study, schizophrenia patients with acute exacerbation received monotherapy with atypical antipsychotic and they showed significant improvements in positive and negative symptoms after 8 weeks of treatment. These results demonstrate a global clinical efficacy of atypical antipsychotics in schizophrenia patients with acute exacerbation. Benefits and harms vary among atypical antipsychotic medications for patients have been reported ${ }^{[25]}$. However, we did not find any significant difference in each PANSS subscore and total score among risperidone, aripiprazole, olanzapine and clozapine-treated groups at the endpoint. This may be because we have just observed the overall clinical effects of antipsychotic drugs on psychiatric symptoms. The analysis of specific symptom item scores would help to further demonstrate the efficiency characteristics of different atypical antipsychotics.

The roles of IGFs in the brain and their potential clinical implications for psychiatric disorders have drawn much attention in recent years ${ }^{[13]}$. The expression of IGFs in the brain is developmentally regulated ${ }^{[27]}$. IGF-1 has the highest expression in perinatal period and decreases in the period of adult. IGF-2 is secreted at high levels during brain development and is the most abundant insulin-like peptide in the adult brain $^{[27,28]}$. Our previous study have shown that serum IGF-2 levels were significantly decreased in patients with schizophrenia, and the levels of serum IGF-2 had a correlation with the severity of negative symptoms ${ }^{[18]}$. Here we found that, in parallel with the improvements of psychiatric symptoms, serum IGF-2 levels in patients after treatment with atypical antipsychotic drugs for two months were significantly increased compared with baseline, and the levels of serum IGF-2 in patients after treatment were comparable with the levels in the controls. These results provide supportive evidence for the neurodevelopmental hypothesis of schizophrenia, and suggest a role for IGF-2 signaling in the pathophysiology of schizophrenia. However, the mechanisms by which the atypical antipsychotic medication induced an increase of serum IGF-2 are unclear. IGF-2 is highly expressed in the choroid plexus, hippocampus, cortex, and leptomeninges ${ }^{[29]}$. The transcription factor CCAAT enhancer binding protein $\beta(\mathrm{C} / \mathrm{EBP} \beta)$ has been shown to regulate the expression of IGF-2 gene $^{[30]}$. Learning could induce an increase of IGF-2 level in the hippocampus by promoting $\mathrm{C} / \mathrm{EBP} \beta$ expression $^{[31]}$. Decrease in C/EPB mRNA levels was observed in patients with schizophrenia ${ }^{[32]}$. Therefore, investigating the influences of atypical antipsychotics on $\mathrm{C} / \mathrm{EBP} \beta$ function would help to elucidate the potential mechanisms underlying IGF-2 elevation caused by atypical antipsychotic treatment in patients.

An interesting finding of current study is that improvement of negative symptoms was significantly correlated to the change of serum IGF-2 levels in patients treated with atypical antipsychotic. This result not only indicates that the serum IGF-2 level could be a peripheral indicator for evaluating the treatment response to atypical antipsychotics, but also suggests that upregulation of IGF-2 signaling might be implicated in the improvement of negative symptoms. Adult severe mental illness largely originates from a disturbed development of the brain during early life ${ }^{[10,33,34]}$. Negative symptoms are an important part of psychopathology in schizophrenia ${ }^{[22]}$. Reduced frontal cortex-striatum functional connectivity had an association with the negative symptom severity in schizophrenia $^{[35]}$. A breakdown of connectivity between the dorsolateral prefrontal cortex and the cerebellum was shown to directly correspond to the severity of negative symptoms, and correction of this breakdown improved the negative symptoms ${ }^{[36]}$. IGF-2 plays an indispensable role in nerve growth ${ }^{[27]}$. It regulates the proliferation, differentiation and maturation of nerve cells including astrocytes, oligodendrocytes, nerve precursor cells and nerve stem cells during the brain development $\mathrm{t}^{[14]}$. Hypomethylation of an enhancer within the IGF-2 gene had been demonstrated in the frontal cortex of schizophrenia patients, and pathway enrichment analysis identified that deletion of IGF-2 enhancer would lead to alterations in cell proliferation, neurodevelopment and cytoskeletal remodeling in the striatum and frontal cortex ${ }^{[16]}$. Thus, we postulated that a beneficial effect of IGF-2 upregulation on abnormal functional connectivity of the brain might contribute to the improvements of negative symptoms in patients treated with atypical antipsychotics. However, this explanation is entirely speculative. Further studies using animal experiments are required to address this presumption.

There are some limitations in this study. First, this is a prospective observational study and selection bias may have been introduced. It should be cautious in generalizing this conclusion beyond the population. Second, we measured the level of IGF-2 in serum. Whether brain IGF-2 levels change parallelly with the alteration of serum IGF-2 is still unknown. Third, the sample size in this study was relatively small. Large-scale clinical studies are needed to replicate and verify this conclusion. Fourth, correlation analysis demonstrates a relationship between negative symptom improvement and serum IGF2 increase in schizophrenia patients. However, the causal relationship between the two variables cannot 
be established. Further study conducted in animal is needed to demonstrate the relationship. Finally, the nutritional status would affect the system of IGFs. Indicators of nutritional conditions, such as the levels of pre-albumin and albumin, should be measured in the subjects.

In conclusion, this study conducted an open-label, prospective observational trial to determine the effects of atypical antipsychotics on psychiatric symptoms and serum IGF-2 levels in schizophrenia patients. The results revealed that atypical antipsychotic medication significantly improved psychiatric symptoms and increased the levels of serum IGF-2 in schizophrenia patients with acute exacerbation. The change in IGF-2 was significantly correlated with the negative symptom improvements, suggesting that regulation of IGF-2 signaling might be implicated in the benefits of atypical antipsychotics on negative symptoms and serum IGF-2 might be a treatment biomarker for schizophrenia.

\section{Open Access}

This article is licensed under a Creative Commons Attribution 4.0 International License https://creativecommons.org/licenses/by/4.0/), which permits use, sharing, adaptation, distribution and reproduction in any medium or format, as long as you give appropriate credit to the original author(s) and the source, provide a link to the Creative Commons licence, and indicate if changes were made. The images or other third party material in this article are included in the article's Creative Commons licence, unless indicated otherwise in a credit line to the material. If material is not included in the article's Creative Commons licence and your intended use is not permitted by statutory regulation or exceeds the permitted use, you will need to obtain permission directly from the copyright holder. To view a copy of this licence, visit http://creativecommons.org/licenses/by/4.0/.

\section{Conflict of Interest Statement}

The authors declare that there is no conflict of interest with any financial organization or corporation or individual that can inappropriately influence this work.

\section{REFERENCES}

1 Owen MJ, Sawa A, Mortensen PB. Schizophrenia. Lancet, 2016,388(10039):86-97

2 Mueser KT, McGurk SR. Schizophrenia. Lancet, 2004, 363(9426):2063-2072

3 Lally J, MacCabe JH. Antipsychotic medication in schizophrenia: a review. Br Med Bull, 2015,114(1):169179

4 Sanchez JPB, Ellenbroek BA. Preclinical Effects of Antipsychotic Drugs. Curr Top Behav Neurosci, 2017, 34:1-16

5 Kapur S, Remington G. Atypical antipsychotics: new directions and new challenges in the treatment of schizophrenia. Annu Rev Med, 2001,52:503-517

6 Muller-Spahn F. Current use of atypical antipsychotics. Eur Psychiatry, 2002, 17 Suppl 4:377s-384s

7 Solmi M, Murru A, Pacchiarotti I, et al. Safety, tolerability, and risks associated with first- and secondgeneration antipsychotics: a state-of-the-art clinical review. Ther Clin Risk Manag, 2017,13:757-777

8 Gallhofer B, Bauer U, Lis S, et al. Cognitive dysfunction in schizophrenia: comparison of treatment with atypical antipsychotic agents and conventional neuroleptic drugs. Eur Neuropsychopharmacol, 1996,6 Suppl 2:S13-20

9 Krakowski MI, Czobor P, Nolan KA. Atypical antipsychotics, neurocognitive deficits, and aggression in schizophrenic patients. J Clin Psychopharmacol, 2008, 28(5):485-493

10 van Os J, Kapur S. Schizophrenia. Lancet, 2009,374 (9690):635-645

11 Zhao C, Zhu J, Liu X, et al. Structural and functional brain abnormalities in schizophrenia: A cross-sectional study at different stages of the disease. Prog Neuropsychopharmacol Biol Psychiatry, 2018,83:27-32

12 Cannon M, Caspi A, Moffitt TE, et al. Evidence for earlychildhood, pan-developmental impairment specific to schizophreniform disorder: results from a longitudinal birth cohort. Arch Gen Psychiatry, 2002,59(5):449-456

13 Benarroch EE. Insulin-like growth factors in the brain and their potential clinical implications. Neurology, 2012,79(21):2148-2153

14 O'Kusky J, Ye P. Neurodevelopmental effects of insulinlike growth factor signaling. Front Neuroendocrinol, 2012,33(3):230-251

15 Fromer M, Roussos P, Sieberts SK, et al. Gene expression elucidates functional impact of polygenic risk for schizophrenia. Nat Neurosci, 2016,19(11):14421453

16 Pai S, Li P, Killinger B, et al. Differential methylation of enhancer at IGF2 is associated with abnormal dopamine synthesis in major psychosis. Nat Commun, 2019,10(1):2046

17 Akanji AO, Ohaeri JU, Al-Shammri SA, et al. Associations of blood levels of insulin-like growth factor (IGF)-I, IGF-II and IGF binding protein (IGFBP)-3 in schizophrenic Arab subjects. Clin Chem Lab Med, 2007,45(9):1229-1231

18 Yang YJ, Luo T, Zhao Y, et al. Altered insulin-like growth factor-2 signaling is associated with psychopathology and cognitive deficits in patients with schizophrenia. PLoS One, 2020,15(3):e0226688

19 Lehman AF, Lieberman JA, Dixon LB, et al. Practice guideline for the treatment of patients with schizophrenia, second edition. Am J Psychiatry, 2004, 161(2 Suppl):1-56

20 Mortimer AM. Antipsychotic treatment in schizophrenia: atypical options and NICE guidance. Eur Psychiatry, 2003,18(5):209-219

21 Peralta V, de Leon J, Cuesta MJ. Are there more than two syndromes in schizophrenia? A critique of the positivenegative dichotomy. Br J Psychiatry, 1992,161:335-343

22 Dollfus S, Lyne J. Negative symptoms: History of the concept and their position in diagnosis of schizophrenia. Schizophr Res, 2017,186:3-7

23 Palmer BW, Dawes SE, Heaton RK. What do we know about neuropsychological aspects of schizophrenia?. Neuropsychol Rev, 2009,19(3):365-384

24 Leucht S, Cipriani A, Spineli L, et al. Comparative efficacy and tolerability of 15 antipsychotic drugs in 
schizophrenia: a multiple-treatments meta-analysis. Lancet, 2013,382(9896):951-962

25 Maher AR, Maglione M, Bagley S, et al. Efficacy and comparative effectiveness of atypical antipsychotic medications for off-label uses in adults: a systematic review and meta-analysis. JAMA, 2011,306(12):13591369

26 Woodward ND, Purdon SE, Meltzer HY, et al. A metaanalysis of neuropsychological change to clozapine, olanzapine, quetiapine, and risperidone in schizophrenia. Int J Neuropsychopharmacol, 2005,8(3):457-472

27 Fernandez AM, Torres-Aleman I. The many faces of insulin-like peptide signalling in the brain. Nat Rev Neurosci, 2012,13(4):225-239

28 Russo VC, Gluckman PD, Feldman EL, et al. The insulin-like growth factor system and its pleiotropic functions in brain. Endocr Rev, 2005,26(7):916-943

29 Logan A, Gonzalez AM, Hill DJ, et al. Coordinated pattern of expression and localization of insulin-like growth factor-II (IGF-II) and IGF-binding protein-2 in the adult rat brain. Endocrinology, 1994,135(5):22552264

30 Shamblott MJ, Leung S, Greene MW, et al. Characterization of a teleost insulin-like growth factor
II (IGF-II) gene: evidence for promoter CCAAT/ enhancer-binding protein $(\mathrm{C} / \mathrm{EBP})$ sites, and the presence of hepatic C/EBP. Mol Mar Biol Biotechnol, 1998,7(3):181-190

31 Chen DY, Stern SA, Garcia-Osta A, et al. A critical role for IGF-II in memory consolidation and enhancement. Nature, 2011,469(7331):491-497

32 Chase KA, Rosen C, Gin H, et al. Metabolic and inflammatory genes in schizophrenia. Psychiatry Res, 2015,225(1-2):208-211

33 Harrison PJ. The neuropathology of schizophrenia. A critical review of the data and their interpretation. Brain, 1999,122 (Pt 4):593-624

34 Han QQ, Shen TT, Wang F, et al. Preventive and Therapeutic Potential of Vitamin C in Mental Disorders. Curr Med Sci, 2018,38(1):1-10

35 Shukla DK, Chiappelli JJ, Sampath H, et al. Aberrant Frontostriatal Connectivity in Negative Symptoms of Schizophrenia. Schizophr Bull, 2019,45(5):1051-1059

36 Brady RO, Jr., Gonsalvez I, Lee I, et al. CerebellarPrefrontal Network Connectivity and Negative Symptoms in Schizophrenia. Am J Psychiatry, 2019, 176(7):512-520

(Received May 20, 2020; revised Jun. 26, 2020) 